\title{
PhysioNet: Physiologic Signals, Time Series and Related Open Source Software for Basic, Clinical, and Applied Research
}

\author{
George B. Moody, Roger G. Mark, and Ary L. Goldberger
}

\begin{abstract}
PhysioNet provides free web access to over 50 collections of recorded physiologic signals and time series, and related open-source software, in support of basic, clinical, and applied research in medicine, physiology, public health, biomedical engineering and computing, and medical instrument design and evaluation. Its three components (PhysioBank, the archive of signals; PhysioToolkit, the software library; and PhysioNetWorks, the virtual laboratory for collaborative development of future PhysioBank data collections and PhysioToolkit software components) connect researchers and students who need physiologic signals and relevant software with researchers who have data and software to share. PhysioNet's annual open engineering challenges stimulate rapid progress on unsolved or poorly solved questions of basic or clinical interest, by focusing attention on achievable solutions that can be evaluated and compared objectively using freely available reference data.
\end{abstract}

\section{INTRODUCTION}

PhysioNet (http://physionet.org/) is a research resource intended to stimulate current research and new investigations in the study of complex biomedical and physiologic signals. It has three major components:

PhysioBank is a large and growing archive of wellcharacterized digital recordings of physiologic signals, time series, and related data for use by the biomedical research community. PhysioBank currently includes more than 50 collections of cardiopulmonary, neural, and other biomedical signals from healthy subjects and patients with a variety of conditions with major public health implications, including sudden cardiac death, congestive heart failure, epilepsy, gait disorders, sleep apnea, and aging (see Tables I and II). These collections include data from a wide range of studies, as developed and contributed by members of the research community.

PhysioToolkit is a large and growing library of software for physiologic signal processing and analysis, detection of physiologically significant events using both classical techniques and novel methods based on statistical physics and nonlinear dynamics, interactive display and characterization of

PhysioNet is supported by the US National Institutes of Health (NIH), its National Institute of Biomedical Imaging and Bioengineering (NIBIB), and its National Institute of General Medical Sciences (NIGMS), under NIH cooperative agreement U01-EB-008577.

GBM and RGM are co-PIs of PhysioNet, and ALG is its program director. GBM is the architect and technical director of PhysioNet, and is with the Laboratory for Computational Physiology (LCP) in the Harvard-MIT Division of Health Sciences and Technology (HST). RGM directs the LCP, and is a Distinguished Professor in HST and EECS at MIT. ALG directs the Rey Institute for Nonlinear Dynamics in Medicine at Boston's Beth Israel Deaconess Medical Center, and is a Professor of Medicine at Harvard Medical School.

Address for correspondence: George B. Moody, Massachusetts Institute of Technology, Room E25-505A, 77 Massachusetts Ave., Cambridge, MA 20139 USA. Email: george@mit .edu signals, creation of new databases, simulation of physiologic and other signals, quantitative evaluation and comparison of analysis methods, and analysis of nonequilibrium and nonstationary processes. A unifying theme of many of the research projects that contribute software to PhysioToolkit is the extraction of "hidden" information from biomedical signals, information that may have diagnostic or prognostic value in medicine, or explanatory or predictive power in basic research. All PhysioToolkit software is available in source form under the GNU General Public License (GPL).

PhysioNetWorks is a virtual laboratory for development of data and software resources that will eventually become components of PhysioBank and PhysioToolkit. By providing large, secure workspaces with redundant backup to active researchers who can easily share them with colleagues anywhere, PhysioNetWorks encourages investigators to create well-organized and documented, usable data and software repositories during the conduct of their research. When the research is complete and the major results have been published (or at any time the researcher wishes) the repository can be shared with a colleague, a group of colleagues, or the research community at large.

\section{BACKGROUND}

PhysioNet was established in 1999 as the outreach component of the Research Resource for Complex Physiologic Signals[1], [2], a cooperative project initiated by the authors at Boston's Beth Israel Deaconess Medical Center, Harvard Medical School, and MIT, together with colleagues at Boston University, McGill University, and later at numerous other institutions. Beginning in the mid-1970s, members of the PhysioNet team who were then working on some of the first microcomputer-based instruments for cardiac arrhythmia monitoring foresaw the usefulness of establishing shared databases of well-characterized ECG recordings, as a basis for evaluation, iterative improvement, and objective comparison of algorithms for automated arrhythmia analysis. A five-year effort culminated in the publication of the MITBIH Arrhythmia Database in 1980, which soon became the standard reference collection of its type, used by over 500 academic, hospital, and industry researchers and developers worldwide during the 1980s and 1990s. Other databases of ECGs and eventually other physiologic signals followed. By 1999, the MIT group distributed CD-ROMs containing 11 such collections, and had participated in the development of several others.

The MIT group contributed its 11 databases, and the software it had developed for exploring and analyzing them, 
to establish PhysioBank and PhysioToolkit. Free availability of these resources via the Internet catalyzed an even greater explosion of interest in them, as researchers and students worldwide who had no previous access to such data or software began new programs of research, and specialists began comparing their methods. These initial contributions were quickly supplemented by additional collections of data and software from their collaborators, and soon after, from many researchers worldwide. PhysioBank and PhysioToolkit have grown to many times their original sizes, and most of the growth has been thanks to the hard work and generosity of an international community of researchers.

\section{ACTIVITIES}

Shortly after PhysioNet was established, we initiated an annual series of open engineering challenges, in cooperation with the annual IEEE-EMBS-sponsored conference, Computers in Cardiology (now Computing in Cardiology, or CinC). We hoped to introduce PhysioNet to our international colleagues who would be attending $\mathrm{CinC}$, by encouraging participation in an activity that made effective use of the facilities provided by PhysioNet to stimulate rapid progress on an unsolved problem of practical clinical significance. A timely contribution of data from Thomas Penzel made it possible to create the first PhysioNet/CinC Challenge, which attracted the attention of more than a dozen teams to the subject of detecting sleep apnea from the ECG[3]. Their efforts were broadly successful, they discussed their findings at CinC 2000, and an annual tradition was born.

In complementary ways, PhysioNet and CinC catalyze and support scientific communication and collaboration between basic and clinical scientists. The annual meetings of CinC are gatherings of researchers from many nations and disciplines, bridging the geographic and specialty chasms that separate understanding from practice, while PhysioNet provides online data and software resources that support collaborations of basic and clinical researchers throughout the year. The annual PhysioNet/CinC Challenges seek to provide stimulating yet friendly competitions, while at the same time offering both specialists and non-specialists alike opportunities to make progress on significant open problems whose solutions may be of profound clinical value.

The use of shared data provided via PhysioNet makes it possible for participants to work independently toward a common objective. At CinC, participants can make meaningful results-based comparisons of their methods; lively and well-informed discussions are the norm at scientific sessions dedicated to these challenges. Discovery of the complementary strengths of diverse approaches to a problem when coupled with deep understanding of that problem frequently sparks new collaborations and opportunities for further study, as occurred when participants in the first Challenge combined their efforts to obtain an even better solution to the Challenge problem[4].
Recent challenge topics have included predicting acute hypotensive episodes in intensive care unit patients[5]; developing robust methods for filling gaps in multiparameter physiologic data (including ECG signals, continuous blood pressure waveforms, and respiration), with applications in detection of clinically important events and in reduction of false alarms in the ICU[6]; and (in progress) improving the quality of ECGs collected using mobile phones.

In a prototype implementation, PhysioNetWorks supported the 2010 Challenge, collecting and scoring entries from the participants. During its first five months (to mid-June 2011) the reimplented PhysioNetWorks has attracted more than 400 members, who are using it to support 12 collaborative projects and 15 others in preparation, in addition to the challenge in progress. Current PhysioNetWorks projects include data collection and annotation development efforts, as well as efforts aimed at improving or evaluating physiologic models and other software projects focused on creation or improvement of tools for research. New contributions of data and software are channeled through PhysioNetWorks, allowing their creators to participate in all aspects of curation of their contributions, and allowing the community to provide early feedback to influence decisions that may affect usability and value to researchers.

Significantly, many PhysioNetWorks projects are being established early in the grant cycles of the associated research projects. Use of PhysioNetWorks throughout the active phase of research encourages investigators to organize their work in a way that makes it easy to use community-developed exploratory and analytic tools by the research team and collaborators in the short run, thus making their final contribution more valuable to the research community at large, and avoiding the pitfalls of attempting to fulfill a mandated data-sharing requirement after funding for the project has ended and those who understand the data have begun work on other projects.

\section{CONCLUSIONS}

In its first 12 years, PhysioNet has made a wide variety and large quantity of well-characterized data and related open-source software collected and created for biomedical research, often at great expense, available for re-use and further study at no cost by a worldwide community of over 40,000 researchers, clinicians, educators and students, and medical instrument and software developers. Through its open engineering challenges, it has stimulated development of inexpensive and minimally disruptive technology for detection of sleep apnea and sleep quality, prediction of adverse events and reduction in false alarms in the intensive care setting, and telemedicine. A Google Scholar search for PhysioNet and related terms finds over 5000 publications and citations as of June 2011. Finally, PhysioNetWorks has allowed us to scale up our capacity to bring new data collections and software packages on-line without requiring a proportional increase in the size of our team. 
TABLE I

PhysioBank Collections of Multiparameter and ECG Signals and Time Series

(AS OF JUNE 2011)

\begin{tabular}{|c|c|c|c|c|}
\hline Collection & Subjects & Duration (typical) & Signals and time series & Other \\
\hline MGH/MF Waveform Database & 250 & $90-120 \mathrm{~min}$ & $\begin{array}{l}\text { ECG (3 leads), ABP, PAP, CVP, } \\
\text { respiration, airway } \mathrm{CO}_{2}, \ldots\end{array}$ & beat annotations \\
\hline Stress Recognition in Automobile Drivers & 17 & $60-90 \mathrm{~min}$ & ECG, EMG, GSR, respiration & \\
\hline Apnea-ECG Database & 70 & 8 hours & ECG (subset includes respiration) & apnea annotations \\
\hline Fantasia Database & 40 & 2 hours & $\begin{array}{l}\text { ECG (subset includes uncalibrated } \\
\text { NIBP) }\end{array}$ & beat annotations \\
\hline MIMIC Database & 121 & 20-40 hours & $\mathrm{ECG}, \mathrm{BP}$, respiration, $\mathrm{SpO}_{2}, \ldots$ & $\begin{array}{l}\text { beat labels, ICU monitor } \\
\text { alarms }\end{array}$ \\
\hline MIMIC II Waveform Database & 20935 & 3-10 days & ECG, BP, respiration, $\mathrm{SpO}_{2}, \ldots$ & \\
\hline MIMIC II Clinical Database & 32536 & 3-10 days & hourly vital signs, medications, ... & $\begin{array}{l}\text { ICD9 codes, lab tests, dis- } \\
\text { charge summaries, ... }\end{array}$ \\
\hline MIT-BIH Polysomnographic Database & 16 & 8 hours & ECG, ABP, EEG, respiration, ... & $\begin{array}{l}\text { apnea and sleep stage annota- } \\
\text { tions }\end{array}$ \\
\hline Sleep-EDF Database & 8 & 8 hours & EEG (2), EOG, ... & hypnograms \\
\hline SVUH/UCD Sleep Apnea Database & 25 & 8 hours & $\begin{array}{l}\text { ECG ( } 3 \text { leads), EEG (2), EOG (2), } \\
\text { EMG, oronasal airflow, ribcage and } \\
\text { abdomen movements, } \mathrm{SpO}_{2} \text {, snor- } \\
\text { ing, body position }\end{array}$ & $\begin{array}{l}\text { apnea and sleep stage annota- } \\
\text { tions }\end{array}$ \\
\hline ANSI/AAMI EC13 Test Waveforms & 10 & 1 minute & ECG & \\
\hline European ST-T Database & 90 & 2 hours & ECG (2 leads) & $\begin{array}{l}\text { beat, rhythm, ST and T change } \\
\text { annotations }\end{array}$ \\
\hline Long-Term ST Database & 86 & 24 hours & ECG ( 2 or 3 leads) & $\begin{array}{l}\text { beat, rhythm, ST and signal } \\
\text { quality annotations }\end{array}$ \\
\hline MIT-BIH Arrhythmia Database & 48 & $30 \mathrm{~min}$ & ECG (2 leads) & $\begin{array}{l}\text { beat, rhythm, and signal qual- } \\
\text { ity annotations }\end{array}$ \\
\hline MIT-BIH Noise Stress Test Database & 15 & $30 \mathrm{~min}$ & ECG (2 leads) with calibrated noise & beat annotations \\
\hline BIDMC Congestive Heart Failure Database & 15 & 20 hours & ECG (2 leads) & beat annotations \\
\hline $\begin{array}{l}\text { Post-Ictal Heart Rate Oscillations in Partial } \\
\text { Epilepsy }\end{array}$ & 7 & $90-220 \mathrm{~min}$ & ECG & beat and seizure annotations \\
\hline QT Database & 100 & $15 \min$ & ECG (2 leads) & $\begin{array}{l}\text { annotations of onsets, peaks, } \\
\text { ends of } \mathrm{P}, \mathrm{QRS} \text {, and } \mathrm{T} \text { waves }\end{array}$ \\
\hline AF Termination Challenge Database & 30 & $1 \mathrm{~min}$ & ECG (2 leads) & beat annotations \\
\hline $\begin{array}{l}\text { Creighton University Ventricular } \\
\text { Tachyarrhythmia Database }\end{array}$ & 35 & $8 \mathrm{~min}$ & ECG (2 leads) & beat and VF annotations \\
\hline Intracardiac Atrial Fibrillation Database & 8 & $3-5 \min$ & $\begin{array}{l}\text { ECG ( } 3 \text { surface and } 5 \text { intracardiac } \\
\text { leads) }\end{array}$ & beat annotations \\
\hline Long-Term AF Database & 84 & 24 hours & ECG (2 leads) & beat annotations \\
\hline MIT-BIH Atrial Fibrillation Database & 25 & 10 hours & ECG (2 leads) & beat and rhythm annotations \\
\hline MIT-BIH ECG Compression Test Database & 168 & $20 \mathrm{sec}$ & ECG (2 leads) & \\
\hline MIT-BIH Long-Term Database & 6 & 24 hours & ECG (2 leads) & beat annotations \\
\hline $\begin{array}{l}\text { MIT-BIH Malignant Ventricular } \\
\text { Arrhythmia Database }\end{array}$ & 22 & $30 \mathrm{~min}$ & ECG (2 leads) & $\begin{array}{l}\text { rhythm and signal quality an- } \\
\text { notations }\end{array}$ \\
\hline MIT-BIH Normal Sinus Rhythm Database & 18 & 24 hours & ECG (2 leads) & beat annotations \\
\hline MIT-BIH ST Change Database & 28 & $20-40 \mathrm{~min}$ & ECG (2 leads) & beat annotations \\
\hline $\begin{array}{l}\text { MIT-BIH Supraventricular Arrhythmia } \\
\text { Database }\end{array}$ & 78 & $30 \mathrm{~min}$ & ECG (2 leads) & beat annotations \\
\hline $\begin{array}{l}\text { Non-Invasive Fetal Electrocardiogram } \\
\text { Database }\end{array}$ & 1 & $5-20 \mathrm{~min}$ & $\begin{array}{l}\text { ECG (maternal and fetal; } 55 \\
\text { recordings over a } 20 \text { week period) }\end{array}$ & maternal beat annotations \\
\hline PAF Prediction Challenge Database & 100 & $30 \min x 2$ & ECG (2 leads) & beat annotations \\
\hline PTB Diagnostic ECG Database & 549 & $2 \min$ & ECG (15 leads) & clinical summaries \\
\hline $\begin{array}{l}\text { St Petersburg INCART 12-lead Arrhythmia } \\
\text { Database }\end{array}$ & 75 & $30 \min$ & ECG (12 leads) & beat annotations \\
\hline Sudden Cardiac Death Holter Database & 23 & 8-24 hours & ECG (2 leads) & beat annotations \\
\hline T-Wave Alternans Challenge Database & 100 & $2 \min$ & ECG (12 leads, some 2 or 3 ) & beat annotations \\
\hline
\end{tabular}


TABLE II

Physiobank Collections of RR Intervals, Gait, Balance, Neuro- and Myoelectric Signals and Time Series

(AS OF JUNE 2011)

\begin{tabular}{|c|c|c|c|c|}
\hline Collection & Subjects & Duration (typical) & Signals and time series & Other \\
\hline CAST RR Interval Sub-Study Database & 809 & 24 hours & - & RR intervals \\
\hline $\begin{array}{l}\text { Congestive Heart Failure RR Interval } \\
\text { Database }\end{array}$ & 29 & 24 hours & - & RR intervals \\
\hline $\begin{array}{l}\text { Exaggerated heart rate oscillations during } \\
\text { two meditation techniques }\end{array}$ & 46 & 10 min - 6 hours & - & RR intervals \\
\hline $\begin{array}{l}\text { Normal Sinus Rhythm RR Interval } \\
\text { Database }\end{array}$ & 54 & 24 hours & - & RR intervals \\
\hline $\begin{array}{l}\text { Spontaneous Ventricular Tachyarrhythmia } \\
\text { Database (Version } 1.0 \text { from Medtronic, } \\
\text { Inc.) }\end{array}$ & 135 & $5-10 \mathrm{~min}$ & - & RR intervals \\
\hline $\begin{array}{l}\text { Gait Dynamics in Neuro-Degenerative } \\
\text { Disease }\end{array}$ & 64 & $2 \min$ & foot pressure & stride intervals \\
\hline Gait in Aging and Disease Database & 15 & $6-15 \min$ & - & stride intervals \\
\hline Gait Maturation Database & 50 & $10 \mathrm{~min}$ & - & stride intervals \\
\hline Gait in Parkinson's Disease & 93 & $5 \min$ & multiple foot force signals & stride intervals \\
\hline $\begin{array}{l}\text { Noise Enhancement of Sensorimotor } \\
\text { Function }\end{array}$ & 27 & 5-10 minutes & postural sway & \\
\hline $\begin{array}{l}\text { Unconstrained and Metronomic Walking } \\
\text { Database }\end{array}$ & 10 & 1 hour & - & stride intervals \\
\hline CHB-MIT Scalp EEG Database & 23 & 1-4 days & 23-26 EEG signals & seizure annotations \\
\hline EEG Motor Movement/Imagery Dataset & 109 & $1-2 \mathrm{~min}$ & 64 EEG signals & task annotations \\
\hline $\begin{array}{l}\text { Effect of Deep Brain Stimulation on } \\
\text { Parkinsonian Tremor }\end{array}$ & 16 & $1 \mathrm{~min}$ & rest tremor velocity & \\
\hline $\begin{array}{l}\text { Evoked Auditory Responses in Normals } \\
\text { across Stimulus Level }\end{array}$ & 8 & $5 \mathrm{~min}$ & $\begin{array}{l}\text { evoked auditory response, oto- } \\
\text { acoustic emissions }\end{array}$ & stimulus annotations \\
\hline Term-Preterm EHG Database & 300 & $30 \mathrm{~min}$ & EHG & \\
\hline Examples of Electromyograms & 3 & $10-30 \mathrm{sec}$ & EMG & \\
\hline
\end{tabular}

\section{REFERENCES}

[1] A.L. Goldberger, L.A.N Amaral, L. Glass, J.M. Hausdorff, P.Ch. Ivanov, R.G. Mark, J.E. Mietus, G.B. Moody, C.-K. Peng, H.E. Stanley. PhysioBank, PhysioToolkit, and PhysioNet: components of a new research resource for complex physiologic signals, Circulation 101(23):e215-e220 [Circulation Electronic Pages; http://circ.ahajournals.org/cgi/content/full/101/23/e215]; 2000 (June 13).

[2] G.B. Moody, R.G. Mark, and A.L. Goldberger. "PhysioNet: a Webbased resource for the study of physiologic signals, IEEE Eng in Med and Biol 20(3):70-75 (May-June 2001).

[3] G.B. Moody, R.G. Mark, A.L. Goldberger, and T. Penzel. Stimulating rapid research advances via focused competition: the Computers in Cardiology Challenge 2000, Computers in Cardiology 27:207-210 (2000).

[4] T. Penzel, J. McNames, P. de Chazal, B. Raymond, A. Murray and G. Moody. Systematic comparison of different algorithms for apnoea detection based on electrocardiographic recordings, Medical \& Biological Engineering \& Computing 40:402-407 (2002).

[5] G.B. Moody, L.H. Lehman. Predicting Acute Hypotensive Episodes: The 10th Annual PhysioNet/Computers in Cardiology Challenge, Computers in Cardiology 36:541-544 (2009). (PMID:20842209)

[6] G.B. Moody. The PhysioNet/Computing in Cardiology Challenge 2010: Mind the Gap, Computing in Cardiology, 37:305308 (2010). 